(type I), 94.7\% (type II), 87.5\% (type III) and 75\% (Jackhammer esophagus/distal esophageal spasm) (IDDF2021-ABS0058 Figure 1. Clinical response to POEM at 5-years followup on intention to treat and per-protocol population) (IDDF2021-ABS-0058 Figure 2. Change in mean Eckardt score at 1 - and 5-years after POEM compared to baseline). Young age was an independent risk factor for the development of dysphagia on follow-up $(\mathrm{p}=0.037)$ (IDDF2021-ABS0058 Table 1). Clinical GERD was detected in $28.6 \%$ of patients. On multivariate analysis, female sex was an independent predictor of symptomatic GERD after POEM $(\mathrm{p}=0.025)$ (IDDF2021-ABS-0058 Table 2).

Conclusions POEM is an effective and durable treatment modality for achalasia and other non-achalasia spastic motility disorders. Young patients and females may be at higher risk for recurrent dysphagia and GERD, respectively.

\section{IDDF2021-ABS-0059 SHORT VERSUS LONG ESOPHAGEAL MYOTOMY DURING PER-ORAL ENDOSCOPIC MYOTOMY (POEM): A SYSTEMATIC REVIEW AND META- ANALYSIS OF COMPARATIVE TRIALS}

'Zaheer Nabi*, ${ }^{1}$ Rupjyoti Talukdar, ${ }^{2}$ Harshal Mandavdhare, ${ }^{1} \mathrm{D}$ Nageshwar Reddy. ${ }^{1}$ Asian Institute of Gastroenterology, India; ${ }^{2}$ Postgraduate Institution of Medical Education and Research, India

\subsection{6/gutjpl-2021-IDDF.124}

Background Per-oral endoscopic myotomy (POEM) is an established modality of treatment for achalasia cardia. Considerable variations exist in the technique of POEM with respect to the length and orientation of the myotomy. In this systematic review and meta-analysis, we compared the outcomes of short versus long myotomy during POEM.

Methods We searched multiple databases from Jan 2010 to March 2021 to identify studies reporting on POEM. We selected studies that reported on comparative outcomes of POEM using short versus long myotomy. We performed a comparative analysis of clinical success, procedural duration, gastroesophageal reflux disease (GERD), and adverse events with short and long myotomy in POEM by metaanalysis.

Results 521 patients from five studies in which 241 patients treated with short and 280 with long myotomy approaches were analyzed. Pooled rate for clinical success gave an odds ratio (OR) of 1.27 (95\% confidence interval [CI] 0.50- 3.26; $\left.I^{2} 0 ; P=0.62\right)$; for hospital stay OR 0.22 (95\%CI $-0.03-$ $\left.0.46 ; I^{2} 0 ; \mathrm{p}=0.08\right)$; for erosive esophagitis was OR 0.58 (95\%CI $\left.0.31-1.07 ; I^{2} 0 ; P=0.08\right)$, and for adverse events was OR 0.67 (95\%CI $\left.0.29-1.53 ; I^{2} 51 ; P=0.34\right)$ (IDDF2021-ABS-0059 Figure 1. Forest plot showing the comparison of clinical success in short versus long myotomy groups) (IDDF2021-ABS-0059 Figure 2. Forest plot showing the comparison of post POEM lower esophageal sphincter pressures in short versus long myotomy groups). Abnormal esophageal acid exposure was less frequent OR $0.45 \quad(95 \%$ CI $0.22-0.90 ; \mathrm{p}=0.02 ; I^{2} 0$ ) (IDDF2021-ABS-0059 Figure 3. Forest plot showing the comparison of post POEM erosive esophagitis in short versus long myotomy groups) and the procedure duration was significantly shorter in the short myotomy group OR $-0.76\left(95 \%\right.$ CI -1.00 to $-0.52 ; I^{2} 43 ; P$ $=0.001)$ (IDDF2021-ABS-0059 Figure 4. Forest plot showing the comparison of procedure duration in short versus long myotomy groups).

Conclusions Short and long myotomy in POEM are comparable to each other in terms of clinical success and adverse

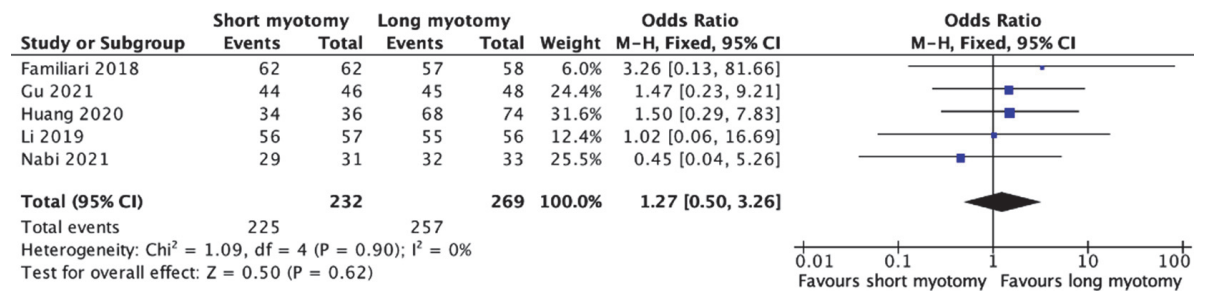

Abstract IDDF2021-ABS-0059 Figure 1

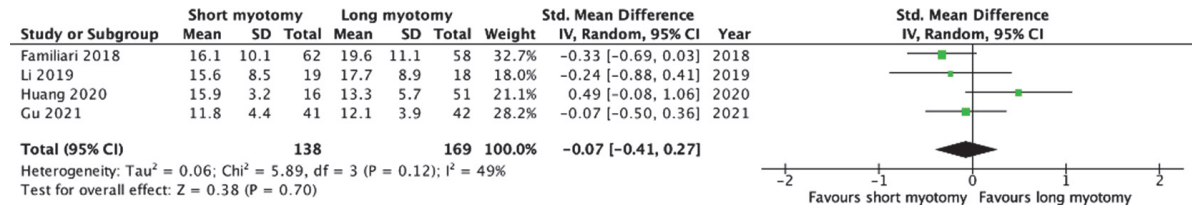

Abstract IDDF2021-ABS-0059 Figure 2

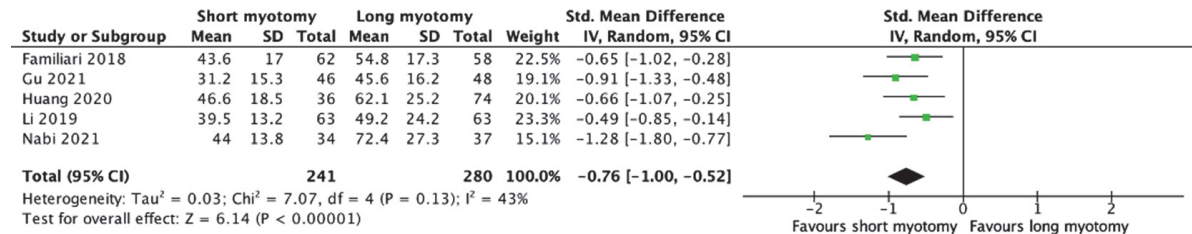




\begin{tabular}{|c|c|c|c|c|c|}
\hline \multirow[b]{2}{*}{ Study or Subgroup } & \multicolumn{2}{|c|}{ Short myotomy } & \multicolumn{2}{|c|}{ Long myotomy } & \multirow[b]{2}{*}{ Weight } \\
\hline & Events & Total & Events & Total & \\
\hline Li, 2019 & 6 & 63 & 8 & 63 & $29.9 \%$ \\
\hline Huang 2020 & 1 & 34 & 4 & 74 & $7.6 \%$ \\
\hline Gu 2021 & 4 & 46 & 7 & 48 & $22.2 \%$ \\
\hline Nabi 2021 & 11 & 34 & 18 & 37 & $40.4 \%$ \\
\hline Total $(95 \% \mathrm{Cl})$ & & 177 & & 222 & $100.0 \%$ \\
\hline Total events & 22 & & 37 & & \\
\hline $\begin{array}{l}\text { Heterogeneity: Tau } \\
\text { Test for overall effe }\end{array}$ & $\begin{array}{l}0.00 ; \mathrm{Chi} \\
=1.76\end{array}$ & $\begin{array}{l}0.24, \\
=0.08)\end{array}$ & $=3(P$ & 97); & $=0$ \\
\hline
\end{tabular}

\section{Abstract IDDF2021-ABS-0059 Figure 4}

events. Short myotomy is associated with significantly shorter procedural duration and possibly reduced reflux as compared to long myotomy.

\section{IDDF2021-ABS-0060 COMPARISON OF CLINICAL OUTCOMES BETWEEN RADIOLOGICAL AND ENDOSCOPIC STRICTURES IN CROHN'S DISEASE: WHICH TYPES OF BOWEL STRICTURES REQUIRE MORE ATTENTION FROM CLINICIAN?}

${ }^{1}$ Li Shi* ${ }^{2}$ Xuehua Li, ${ }^{3}$ Ren Mao, ${ }^{2}$ Canhui Sun, ${ }^{2}$ Ziping Li, ${ }^{2}$ Shiting Feng. ${ }^{1}$ Department of Radiology, The Third Affiliated Hospital of Guangzhou Medical University, Guangzhou, China; ${ }^{2}$ Department of Radiology, The First Affiliated Hospital of Sun Yat-Sen University, Guangzhou, China; ${ }^{3}$ Department of Gastroenterology, The First Affiliated Hospital of Sun Yat-Sen University, Guangzhou, China

\subsection{6/gutjnl-2021-IDDF.125}

Background The definition of 'bowel stricture' in Crohn's disease (CD) is not uniform across studies, including radiological strictures (RS) and endoscopic strictures (ES). Few studies reported the differences of clinical outcomes between RS and ES in CD. Moreover, whether upstream dilatation (UD) is necessary to diagnosis RS remains unknown. We aimed to compare the clinical outcomes between RS and ES, for exploring which type of stricture requires more attention from clinicians and the significance of UD in RS.

Methods This retrospective study enrolled 431 strictures which simultaneously underwent endoscopy and radiologic examinations. ES was defined as an endoscopic non-passable stricture; RS was defined as a luminal narrowing (at least $50 \%$ decrease) with wall thickening (at least $25 \%$ increase) relative to adjacent normal gut on CT/MRI. The strictures were categorized as Group 1 (G1, only met RS definition; further divided into G1a [without UD] or G1b [with UD]), Group 2
Odds Ratio H, Random, 95\% Cl Year $0.72[0.24,2.22] 2019$ $0.53[0.06,4.93] 2020$ $0.56[0.15,2.05] 2021$ $0.50[0.19,1.33] 2021$

$0.58[0.31,1.07]$

\section{$0.58[0.31,1.07]$}

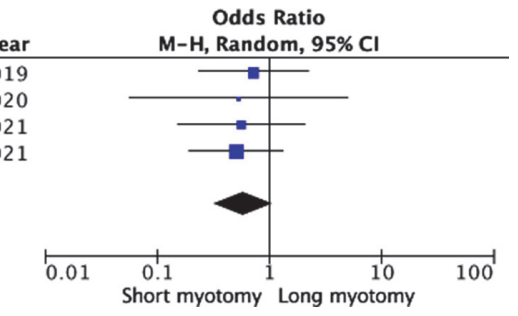

Odds Ratio

(G2, only met ES definition), or Group 3 (G3, met the definitions of RS and ES). Clinical adverse outcomes (CAO) referred to stricture-related surgery or penetrating diseases. The follow-up endpoint was the time occurring CAO or November 30, 2020, if absent of CAO.

Results G1b (65.0\%) had the highest occurrence rate of CAO, followed by G3 (40.4\%), G1a (12.6\%), and G2 (4\%) $(P<0.0001)$. Using Kaplan-Meier curves analysis, the time distribution of occurring $\mathrm{CAO}$ among these groups also had significant differences $(P<0.0001$; IDDF2021-ABS-0060 Figure 1). In $\mathrm{G} 1, \mathrm{G} 1 \mathrm{~b}$ had a higher occurrence rate of $\mathrm{CAO}$ than G1a $(P<0.0001)$. UD (HR 2.082; 95\% CI, 1.651-2.628) was a risk factor for predicting $\mathrm{CAO}$ in multivariate analysis; after adding it into radiologic model, UD significantly improved the prediction efficiency of the model (integrated discrimination improvement, $13.30 \%, 13.20 \%, 9.90 \%$ in 52, 156, 260 weeks, respectively; IDDF2021-ABS-0060 Figure 2). However, if adding UD to diagnose RS, 40 high-risk strictures would be unnoticed.

Conclusions The CAO is different between ES and RS; clinicians should pay more attention for strictures in G1b and G3. UD has an important impact on the clinical outcome of RS but may not an essential factor for RS diagnosis.

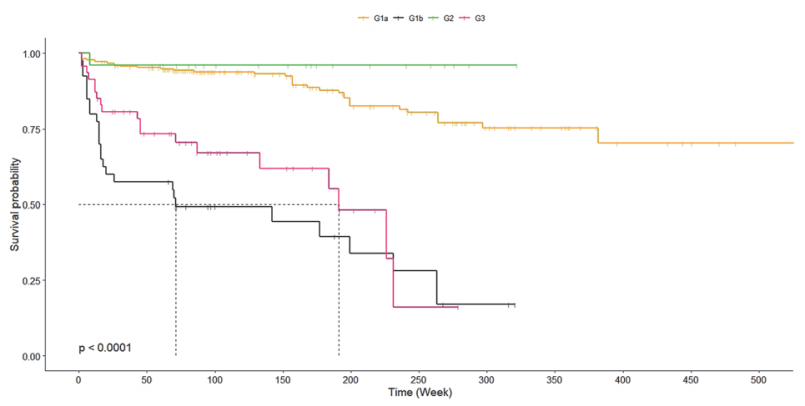

Abstract IDDF2021-ABS-0060 Figure 1
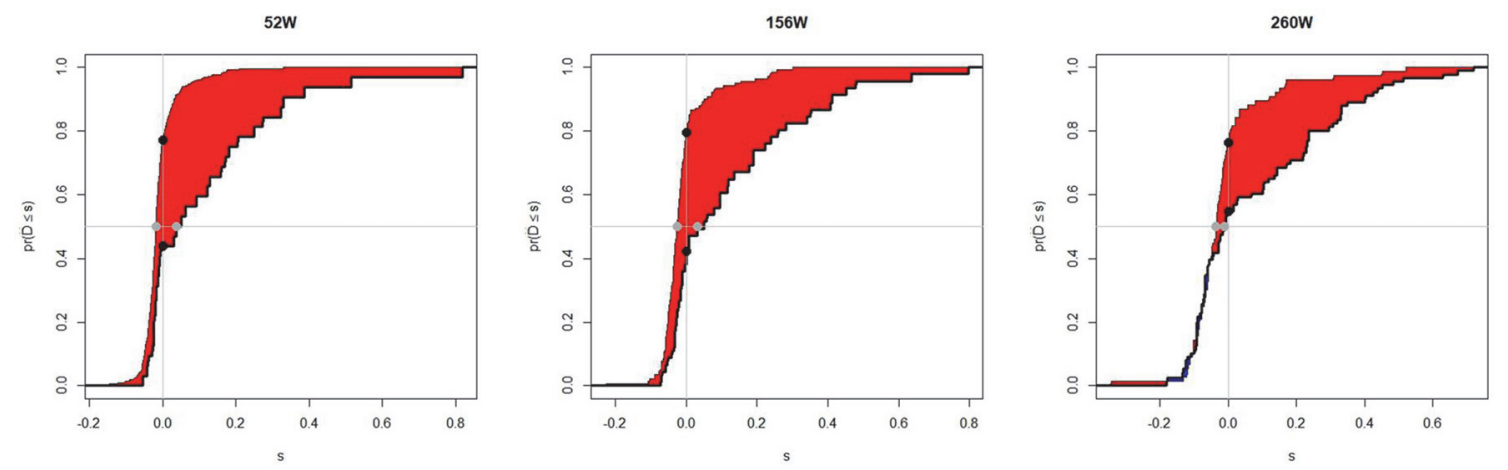

Abstract IDDF2021-ABS-0060 Figure 2 